\title{
African penguins follow the gaze direction of conspecifics
}

\author{
Christian Nawroth ${ }^{\text {Corresp.. }}{ }^{1,2}{ }^{\text {, Egle Trincas }}{ }^{3,4}$, Livio Favaro $^{3}$ \\ ${ }^{1}$ School of Biological and Chemical Sciences, Queen Mary University of London, London, United Kingdom \\ 2 Institute of Behavioural Physiology, Leibniz Institute for Farm Animal Biology, Dummerstorf, Germany \\ 3 Department of Life Sciences and Systems Biology, University of Turin, Turin, Italy \\ 4 Zoom Torino, Turin, Italy \\ Corresponding Author: Christian Nawroth \\ Email address: nawroth.christian@gmail.com
}

Gaze-following is widespread among animals. However, the corresponding ultimate functions may vary substantially. It is thus important to study previously understudied (or less studied) species in order to develop a better understanding of the ecological contexts that foster certain cognitive traits. Penguins (Family Spheniscidae), despite their wide inter-species ecological variation, have previously not been considered for cross-species comparisons. Penguin behaviour and communication have been investigated over the last decades, but less is known on how groups are structured, social hierarchies are established, and coordination for hunting and predator avoidance may occur. We here investigated how African penguins (Spheniscus demersus) respond to gaze cues of conspecifics using a naturalistic setup in a zoo environment. Our results provide evidence that members of the family Spheniscidae follow gaze of conspecifics into distant space. However, further tests are necessary to examine if the observed behaviour serves solely one specific function (e.g. predator detection) or is displayed in a broader context (e.g. eavesdropping on relevant stimuli in the environment). In addition, our findings can serve as a starting point for future cross-species comparisons with other members of the penguin family, in order to further explore the role of aerial predation and social structure on gaze following in social species. Overall, we also suggest that zoo-housed animals represent an ideal opportunity to extend species range and to test phylogenetic families that have not been in the focus of animal cognitive research. 


\section{African penguins follow the gaze direction of conspecifics}

2

3 Christian Nawroth ${ }^{1,2}$, Egle Trincas ${ }^{3,4}$, Livio Favaro 3

4

$5{ }^{1}$ Queen Mary University of London, School of Biological and Chemical Sciences, London, UK

62 Leibniz Institute for Farm Animal Biology, Institute of Behavioural Physiology, Dummerstorf, GER

$7 \quad 3$ University of Turin, Department of Life Sciences and Systems Biology, Turin, IT

$8 \quad 4$ Zoom Torino, Cumiana, Turin, IT

9

10

11

12

13

14

15

16

17

18 Corresponding author

19 Christian Nawroth

20 Email address: nawroth.christian@gmail.com 


\section{Abstract}

23

24 Gaze-following is widespread among animals. However, the corresponding ultimate functions may vary 25 substantially. It is thus important to study previously understudied (or less studied) species in order to develop a better understanding of the ecological contexts that foster certain cognitive traits. Penguins

27 (Family Spheniscidae), despite their wide inter-species ecological variation, have previously not been considered for cross-species comparisons. Penguin behaviour and communication have been investigated over the last decades, but less is known on how groups are structured, social hierarchies are established, and coordination for hunting and predator avoidance may occur. We here investigated how African penguins (Spheniscus demersus) respond to gaze cues of conspecifics using a naturalistic setup in a zoo environment. Our results provide evidence that members of the family Spheniscidae follow gaze of conspecifics into distant space. However, further tests are necessary to examine if the observed behaviour serves solely one specific function (e.g. predator detection) or is displayed in a broader context (e.g. eavesdropping on relevant stimuli in the environment). In addition, our findings can serve as a starting point for future cross-species comparisons with other members of the penguin family, in order to further explore the role of aerial predation and social structure on gaze following in social species. Overall, we also suggest that zoo-housed animals represent an ideal opportunity to extend species range and to test phylogenetic families that have not been in the focus of animal cognitive research. 


\section{Introduction}

42

43 Gaze-following is widespread in the animal kingdom and has been reported for a variety of nonhuman 44 primates (Rosati \& Hare, 2009), but also other mammals (Kaminski et al., 2005; Werhahn et al., 2016), birds (Schloegl, Kotrschal \& Bugnyar, 2007), and reptiles (Wilkinson et al., 2010). However, although several species share this skill, the corresponding ultimate functions may vary and at least three different cognitive mechanisms have been classified: Gaze following into distant space, geometrical gaze following and shared attention (Emery, 2000; Davidson et al., 2014). The first is defined as the co-orientation of one's own gaze direction into distant space with that of another individual (Tomasello, Call \& Hare, 1998; Wilkinson et al., 2010). This form of visual co-orientation is an automatic, reflexive shift of gaze in response to another individual gazing to search for anything interesting along this line of sight (Gómez, 2005). On the other hand, geometric gaze following enables a subject to follow the gaze of another individual around barriers - an ability that would require a subject to take the visual perspective of another individual (Bräuer, Call \& Tomasello, 2005). A third form of following the gaze direction of another individual is to share attention towards an external focal object or event (Gómez, 2005).

Investigations on gaze cues have been a major focus in primate research (Rosati \& Hare, 2009). For instance, Great apes and several monkey species know what conspecifics or humans can see (Hare et al., 2000; Bulloch, Boysen \& Furlong, 2008; Botting, Wiper \& Anderson, 2011; Overduin-de Vries, Spruijt \& Sterck, 2013). This is not surprising, given that animals that live in groups would benefit from cognitive skills that allow them to monitor and eavesdrop on other individuals, and would thus be able to better compete with others over critical resources (Kummer et al., 1997). Recently, social structure and group size in primates have been linked to the interpretation of gaze cues (Tomasello, Call \& Hare, 1998; MacLean et al., 2013). For example, species of lemurs living in larger groups followed the gaze of an experimenter into distant space more often than lemurs from species that have less complex social systems (Sandel, MacLean \& Hare, 2011). 
68 In birds, most work on gaze cues and gaze following has been done on members of the corvid family (Seed, 69 Emery \& Clayton, 2009; von Bayern \& Emery, 2009). It has been found that ravens (Corvus corax) follow 70 the gaze of conspecifics into distant space (Bugnyar, Stöwe \& Heinrich, 2004; Schloegl, Kotrschal \& 71 Bugnyar, 2007), but are also able to follow gaze around barriers (Bugnyar, Stöwe \& Heinrich, 2004). Recently, other ,non-corvid, birds such as Northern bald ibises, Geronticus eremita (Loretto, Schloegl \& Bugnyar, 2010), Greylag geese, Anser anser (Kehmeier et al., 2011), and European starlings, Sturnus vulgaris (Butler \& Fernandez-Juricic, 2014) have also been found to follow gaze of conspecifics. Gaze following in altricial birds, such as corvids, seems to emerge shortly after fledging (Schloegl, Kotrschal \& Bugnyar, 2007) - a time when individuals will experience higher exposure to aerial predation. The so-called 'predator hypothesis' states that gaze following into distant space, at least in birds, might be a protective measure against predators. In turn, we would expect that species exposed to high levels of aerial predation should be more prone to follow the gaze of others into distant space than subjects with lower predation risks (Gómez, 2005). However, there are no studies available that directly link risk of predation and individual's inclination to follow gaze. Thus, more genera and species are required in comparative cognition research in order to develop a better understanding of the ecological contexts that foster certain cognitive traits and to track their evolutionary origins (Shettleworth, 2009).

Penguins (Aves, Spheniscifomes, Spheniscidae) are a family of seabirds that have evolved to swim and have lost the ability to fly (Williams, 1995). The common ancestry of penguins dates back to about 40 mya and the family is now divided into six extant genera (Baker et al., 2006). Over the last decades, penguin behaviour gained attention, particularly in terms of their abilities to differentiate conspecifics using their vocalisations for relocating mates and offspring in densely packed groups (Aubin, Jouventin \& Hildebrand, 2000; Clark, Boersma \& Olmsted, 2006). However, less is known on how groups are structured and social hierarchies are established (but see Ancel et al., 2015). There are also no studies available that investigate the use of gaze cues in social contexts (e.g. during foraging or predator detection/avoidance). We here tested African penguins about their ability to respond to gaze cues of conspecifics in a semi-naturalistic setting. African penguins are social animals, including a complex system of vocal communication (Favaro, 
96 (Pichegru, 2013) - both factors that are considered to affect the interpretation of gaze cues from 97 conspecifics (Gómez, 2005; Sandel, MacLean \& Hare, 2011; MacLean et al., 2013). Given the exposure of 98 the African penguin to such ecological sources of selection, we expected this species to be able to co-orient 99 with conspecifics' gaze.

100

101

\section{Methods}

102

103

\section{Ethical Note}

The research was carried out with permission from Zoom Torino S.p.A. (www.zoomtorino.it). This zoological institution has high standards for animal welfare and is accredited by the EAZA (European Association of Zoos and Aquaria) and UIZA (Unione Italiana Giardini Zoologici e Acquari). Because all recording procedures were non-invasive and did not cause any disturbance to the animals, the approval of an ethics committee was not required by Italian laws. Animal care and all experimental procedures were in accordance with the ASAB/ABS Guidelines for the Use of Animals in Research (Association for the Study of Animal Behaviour, 2016).

111

\section{Subjects and housing}

113 A total of 45 African penguins were tested (21 males, 24 females), aged 1-29 years (mean \pm SEM: $8.2 \pm$ 114 1.1). The birds were all members of a large ex-situ colony (i.e., 64 individuals on October 2016) hosted in 115 a semi-naturalistic enclosure $\left(1500 \mathrm{~m}^{2}\right.$ and a swimming pool of $\left.120 \mathrm{~m}^{2}\right)$ at the biopark Zoom Torino, 116 Cumiana (TO), Italy $\left(44^{\circ} 56^{\prime} \mathrm{N}, 7^{\circ} 25^{\prime} \mathrm{E}\right)$. Daily care of the animals was provided by zoo employees and 117 volunteers.

\section{Experimental procedure}

120 All the tests were carried out in September and October 2016 (i.e., a period where the impact of the viewing 121 public is minimal to the study group; Ozella et al., 2015). The experiment was conducted by a single human

122 (E.T.) who was equipped with a laser pointer and was standing outside the penguin enclosure (similar to 123 an experiment on Norther bald ibises: Loretto, Schloegl \& Bugnyar, 2010). In test trials (T), the experimenter 
124 waited for the occurrence of spatial configurations in which one penguin (the test subject) was facing 125 towards the experimenter, and another subject (the demonstrator) was in a position where it is in front of 126 the test subject with its back turned towards the experimenter (Figure 1). To control for stimulus and local 127 enhancement, the spatial configuration had to be as follows: demonstrator - test subject - laser dot (Figure 128 1). The experimenter was projecting for a maximum of $3 \mathrm{~s}$ (or as soon as the demonstrator looked towards) 129 a laser dot onto a close obstacle (e.g., a wall or a wooden fence) and scored the behaviour of the test 130 subject for the following 5s. Because penguins show vigilance behaviour while resting (i.e., 131 observing/screening their environment), we administered a first control condition (C1) to exclude for 132 accidental gaze co-orienting between two subjects. The initial constellation of the two subjects 133 (demonstrator and test subject) was identical as in test trials, but no laser dot was presented. An additional second control condition (C2) was administered to exclude the possibility that the tested subjects changed 135 behaviour because they responded to the laser dot instead of the change in gaze direction of the demonstrator. In this control condition, no demonstrator was present, and the experimenter was projecting for 3 s a laser dot onto a close obstacle (e.g., wall or wooden fence) behind the test subject and scored its behaviour for the following $5 \mathrm{~s}$. The distance between demonstrator and test subject in $\mathrm{T}$ and $\mathrm{C} 1$ trials varied (approximately 5-20m), as several potential spatial locations in the enclosure were used. This was also the case for the distance between the experimenter and the demonstrator or test subject, which, depending on the position of the animals in the enclosure, varied as well (approximately $0.5-4 \mathrm{~m}$ ). Some subjects were

142 tested more than once due to the opportunistic nature of the test design and received trials of each condition 143 in a random and opportunistic order. This resulted in the fact that demonstrator and test subject were not 144 necessarily the same in T and C1 trials. However, subjects received a maximum of one trial an hour. Each 145 bird had flipper bands for individual recognition and the experimenter (E.T.) reliably identified individuals because of previous experience in handling them. 


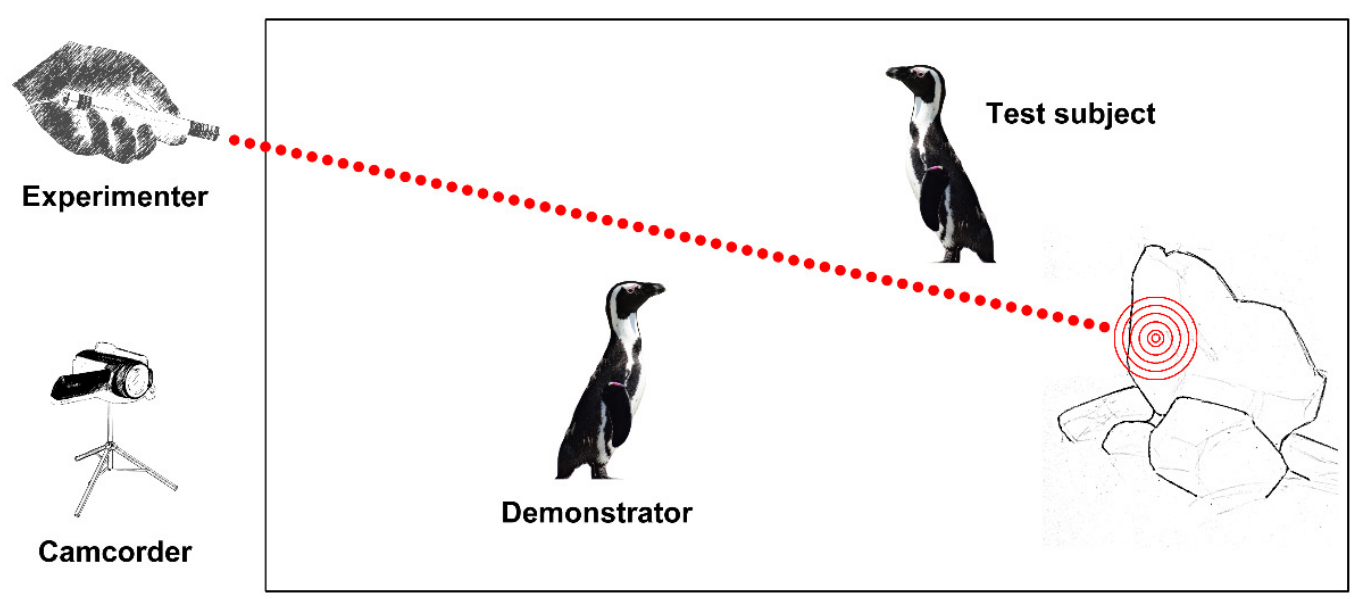

149 Figure 1. Experimental setup for test ( $T$ ) and control (C1) trials. The 'test subject' had to be oriented towards 150 the experimenter, while the demonstrator had to be positioned between experimenter and test subject. 151 Additional control trials (C2) looked identical, but without a demonstrator subject.

Data coding and statistical analysis

154 All trials were videotaped from the experimenter's position using a Sony HDR-SR5E HD camcorder. 155 Subjects' responses in trials were scored via video analysis using BORIS (Behavioral Observation 156 Research Interactive Software (Friard \& Gamba, 2016)). For the test condition and C2, we analysed 157 whether the test subject changed gaze and was looking in the same direction where the laser dot was 158 presented within a maximum of $8 \mathrm{~s}$ ( $3 \mathrm{~s}$ dot presentation $+5 \mathrm{~s}$ additional response time without dot) after a 159 trial started. For $\mathrm{C} 1$, we chose a 10s sequence of the test subject and the demonstrator and screened the entire sequence for gaze-orienting behaviour by the focal test subject. As T and C1 trials needed the same spatial configuration of demonstrator and test subject, the experimenter randomly assigned specific situation to either T or $\mathrm{C} 1$ trials. For $\mathrm{T}$ trial analysis, we also included all trials in which the demonstrator did not look towards the laser dot after presentation, because we could not exclude that the demonstrator might have expressed more subtle head orientation cues that could not have been easily spotted by the experimenter. We used beak direction of test subjects to assess the direction in which penguins gazed. Test subjects had to move their head at least 45 degrees and duration of movement had to be below 1 
167

168

169

170

171

172

173

174

175

176

177

178

179

180

181

182

183

184

185

186

187

188

189

190

191

192

193

194

second. Using this approach, we could avoid including vigilance behaviour, which usually involves slower movements of the head. Penguins hunt fish and marine invertebrates (Williams, 1995), so we expected them to use binocular vision when they have to focus on specific objects or events (Martin, 2009). We thus assume that beak orientation is a reliable measure of attention for this species. A substantial percentage of the trials ( $>40 \% ; 57 / 129$ trials) was randomly chosen and was then coded by a second observer. Interobserver reliability for gaze-orienting behaviour was very good (Cohen's $\mathrm{k}=0.879$ ). A Generalised Linear Mixed Model (GLMM) with binomial logit link using the function glmer of the R package Ime4 was used for analysis. Test subject response (i.e., looking towards the target location) was binary scored with either 'yes' or 'no' and was used response variable. Because we opportunistically had to choose potential pair settings, total trial number of subjects ranged between 1-7 trials in total (mean \pm SEM: $2.867 \pm 0.273$ ), leading to a total of $49 \mathrm{~T}$ trials, $46 \mathrm{C} 1$ trials, and $33 \mathrm{C} 2$ trials. Test condition, age and sex of the tested subjects were included as fixed factors, while identity of tested subjects and demonstrator subjects was included as random factor, thus taking into account repeated measures for individual subjects. Before running the models, we excluded the occurrence of collinearity among predictors by examining the variance inflation factors (vif package; Fox \& Weisberg, 2011). All the predictors showed vif $<2$.

\section{Results}

We analysed a total of 49 test trials ( $T$ ) and 46 control trials (C1). In T trials, the demonstrator subjects looked towards the laser dot in $44 / 49$ trials ( $89.8 \%$ of all test trials). We found a significant effect of condition (GLMM: $X^{2}=11.658 ; d f=1 ; P<0.001$ ) on penguins' response rate between $\mathrm{T}$ and $\mathrm{C} 1$ trials (Figure 2). Subjects co-oriented with the gaze direction of a conspecific significantly more often in the test than in the control condition (T vs. C1: $24.5 \%$ vs $2.1 \%$ of the trials; see ESM video). We found no effect of sex (GLMM: $\left.X^{2}=2.146 ; d f=1 ; P=0.143\right)$ or age $\left(G L M M: X^{2}=2.284 ; d f=1 ; P=0.131\right)$ on gazing behaviour. Additional controls trials $(\mathrm{C} 2 ; \mathrm{N}=33)$ were conducted to exclude that test subjects' behavioural change was due to following the gaze direction of conspecifics and not because they perceived the laser dot behind them in T trials. In none of the $\mathrm{C} 2$ trials did the birds turn their gaze towards the laser dot ( $0 \%$ of trials). Because none of the subjects responded in $\mathrm{C} 2$ trials, we did not include these trials in the GLMM model. 


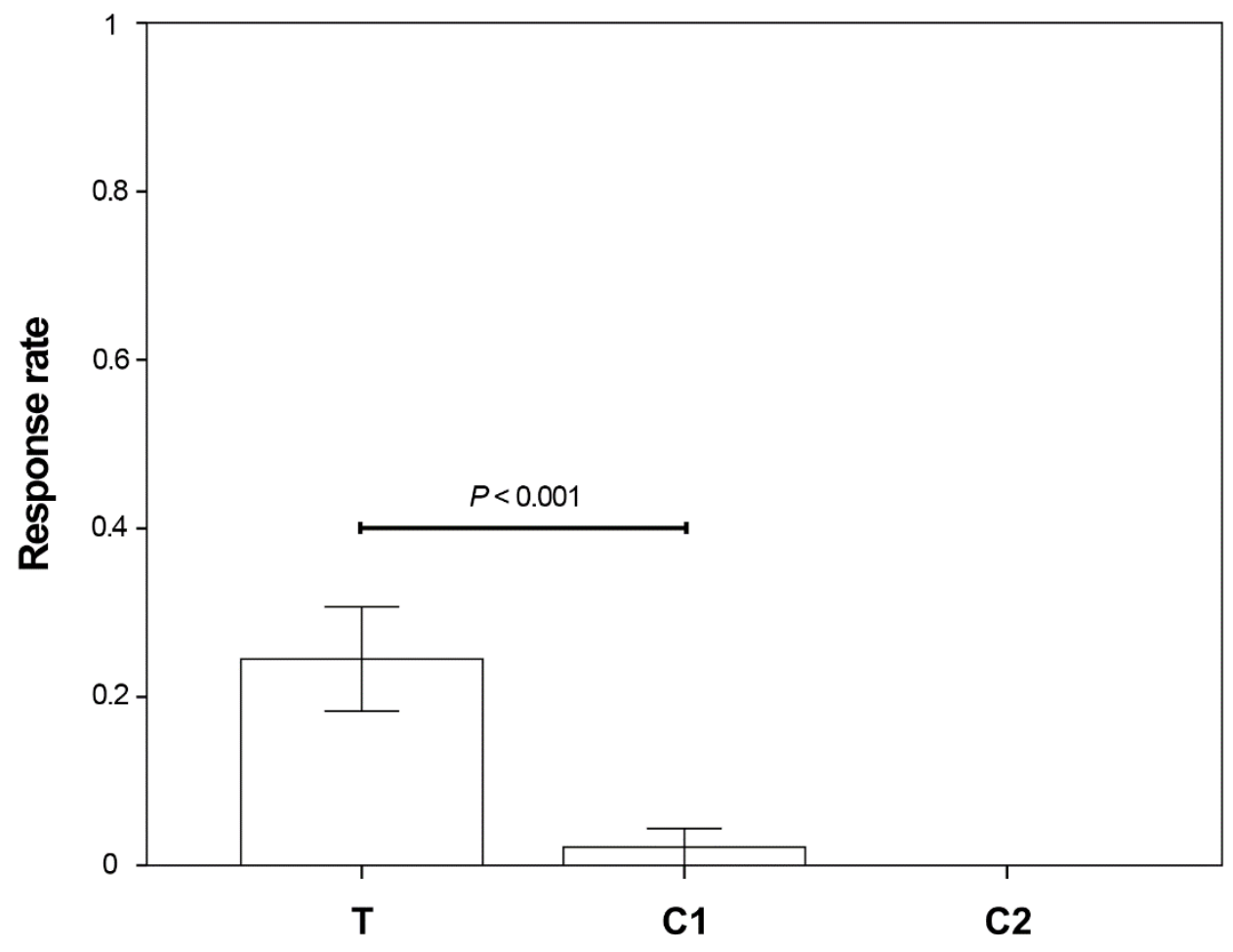

Figure 2 Relative response rate of test subjects for the three different test conditions $\left(T_{\text {subjects }}=33, \mathrm{C} 1\right.$ subjects $=29, \mathrm{C} 2$ subjects $=18 ; \mathrm{T}_{\text {trials }}=49, \mathrm{C} 1_{\text {trials }}=46, \mathrm{C} 2$ trials $=33$ ). Bars represent mean $\pm \mathrm{SE}$.

Discussion

201

We investigated the ability of penguins to follow the gaze direction of conspecifics using a semi-naturalistic setup in a zoo environment. Our results provide evidence that African penguins co-orient with the gaze of other penguins, while we ruled out that inadvertent cueing and spontaneous co-orienting account for our results.

Penguins in our experiment followed the gaze of conspecifics in $24.5 \%$ of the test trials. This is a striking difference to the relative absence of the same behaviour in our control conditions, but can be considered a rather small rate when compared with the response rate in other bird species. For example, young ravens showed a positive response in $40 \%$ of test trials (Schloegl, Kotrschal \& Bugnyar, 2007), while positive 
211 response rate for Northern bald ibises was above 60\% (Loretto, Schloegl \& Bugnyar, 2010). One

212 explanation for these differences might be a rather low inclination of African penguins to follow gaze of

213 conspecifics. Alternatively, environmental cues might have distracted test subjects. Subjects were tested

214 in their home enclosure, exposing them to potential visual, but also acoustic, cues from a large number of

215 conspecifics. Environmental noise and movement might have further diverted subjects' attention. Finally,

216 penguins have laterally positioned eyes, so we cannot exclude the possibility that penguins might perceive

217 the head movement of the demonstrator without necessarily co-orienting their head in the same line.

218 However, penguins hunt fish and marine invertebrates (Williams, 1995), so we would expect them to use

219 binocular vision when they have to focus on specific objects or events (Martin, 2009).

220

221

As our study subjects were housed in a zoo and thus have frequent experience with humans, it would be intriguing too know whether they would also follow gaze cues by humans (Bugnyar, Stöwe \& Heinrich, 2004; Schloegl, Kotrschal \& Bugnyar, 2007). Unfortunately, we were not able to test this as the presence

of humans in the animals' enclosure led to huddling behaviour which did not allow us to test subjects individually.

Different penguin species experience, depending on their geographical location, different degrees of aerial predation (Young, 2005). As the only penguin species that lives on the African continent, the African penguin breeds on islands and coastal areas of South Africa and Namibia and is exposed to aerial predators like the kelp gull (Larus dominicanus) and other birds of prey targeting their eggs and chicks (Pichegru, 2013). In contrast, emperor penguins (Aptenodytes forsteri) should be relatively safe from aerial predation due to the harsh climate which they choose for breeding. This makes the penguin family (Spheniscidae) an ideal model taxon to investigate hypotheses that refer to gaze following into distant space as a skill to increase the chances of an individual to detect aerial predators (Gómez, 2005). The African penguin is also a social species, nesting in dense colonies, with a complex system of vocal communication (Favaro, Ozella \& Pessani, 2014; Favaro et al., 2015). In primates, gaze following has been linked to social structure and group size (Tomasello, Call \& Hare, 1998; Sandel, MacLean \& Hare, 2011) and it would be of interest 
size as well.

240

241 Our study design did not allow us to differentiate whether penguins used gaze following for predator detection. Against this hypothesis, some subjects even walked towards the place where the demonstrator has seen the laser dot (see ESM video) - a behaviour which is certainly not adaptive in the context of predator detection. It is thus likely that penguins in our experiment used gaze cues of demonstrators to access general information from the environment. A few demonstrators also, after gazing, oriented their full body towards the laser dot, and thus some subjects might have used full body orientation rather than gaze only to co-orient with conspecifics. Thus, the observed behaviour is more likely to be used for group coordination in penguins, e.g. in the context of foraging or movement initiation (Briard, Dorn \& Petit, 2015).

Overall, our results provide evidence that penguins follow gaze of conspecifics. This paves the way for

future cross-species comparisons that will further our understanding of the behavioural mechanisms foraging at sea (Sutton, Hoskins \& Arnould, 2015). We also suggest that cognitive testing of species housed ex-situ that have not been established in animal cognition research are a promising way to highlight ultimate functions of a diverse set of mental traits in non-human animals (Hopper, 2017).

\section{Acknowledgments}

We thank the staff from Zoom Torino for their excellent help and free access to the animals, in particular Daniel Sanchez and Valentina Isaja. We would like to thank Marie-Sophie Single for help during video analysis. We are grateful to Luigi Baciadonna for advising on statistical analysis and providing comments a previous version of the manuscript.

262

\section{References}

Ancel A., Gilbert C., Poulin N., Beaulieu M., Thierry B. 2015. New insights into the huddling dynamics of 266 emperor penguins. Animal Behaviour 110:91-98. DOI: 10.1016/j.anbehav.2015.09.019. 
267 268

Association for the Study of Animal Behaviour. 2016. Guidelines for the treatment of animals in behavioural research and teaching. Animal Behaviour 111:I-IX.

Aubin T., Jouventin P., Hildebrand C. 2000. Penguins use the two-voice system to recognize each other. Proceedings of the Royal Society of London B: Biological Sciences 267:1081-1087. DOI: 10.1098/rspb.2000.1112.

Baker AJ., Pereira SL., Haddrath OP., Edge K-A. 2006. Multiple gene evidence for expansion of extant penguins out of Antarctica due to global cooling. Proceedings of the Royal Society of London B: Biological Sciences 273:11-17.

von Bayern AMP., Emery NJ. 2009. Jackdaws respond to human attentional states and communicative cues in different contexts. Current Biology 19:602-6. DOI: 10.1016/j.cub.2009.02.062.

Botting JL., Wiper ML., Anderson JR. 2011. Brown (Eulemur fulvus) and ring-tailed lemurs (Lemur catta) use human head orientation as a cue to gaze direction in a food choice task. Folia Primatologica 82:165-176. DOI: 10.1159/000333142.

Bräuer J., Call J., Tomasello M. 2005. All great ape species follow gaze to distant locations and around barriers. Journal of Comparative Psychology 119:145-54. DOI: 10.1037/0735-7036.119.2.145.

Briard L., Dorn C., Petit O. 2015. Personality and Affinities Play a Key Role in the Organisation of Collective Movements in a Group of Domestic Horses. Ethology 121:888-901. DOI: 10.1111/eth.12402.

Bugnyar T., Stöwe M., Heinrich B. 2004. Ravens, Corvus corax, follow gaze direction of humans around obstacles. Proceedings of the Royal Society of London B: Biological Sciences 271:1331-1336. DOI: 10.1098/rspb.2004.2738.

Bulloch MJ., Boysen ST., Furlong EE. 2008. Visual attention and its relation to knowledge states in chimpanzees, Pan troglodytes. Animal Behaviour 76:1147-1155. DOI: 10.1016/j.anbehav.2008.01.033.

Butler SR., Fernandez-Juricic E. 2014. European starlings recognize the location of robotic conspecific attention. Biology Letters 10:20140665-20140665. DOI: 10.1098/rsbl.2014.0665.

Clark JA., Boersma PD., Olmsted DM. 2006. Name that tune: call discrimination and individual recognition in Magellanic penguins. Animal Behaviour 72:1141-1148. DOI: 
10.1016/j.anbehav.2006.04.002.

296

297

298

299

300

301

302

303

304

305

306

307

308

309

310

311

312

313

314

315

316

317

318

319

320

321

322

Davidson GL., Butler S., Fernández-Juricic E., Thornton A., Clayton NS. 2014. Gaze sensitivity: function and mechanisms from sensory and cognitive perspectives. Animal Behaviour 87:3-15. DOI: 10.1016/j.anbehav.2013.10.024.

Emery NJ. 2000. The eyes have it: the neuroethology, function and evolution of social gaze. Neuroscience and biobehavioral reviews 24:581-604.

Favaro L., Gamba M., Alfieri C., Pessani D., McElligott AG. 2015. Vocal individuality cues in the African penguin (Spheniscus demersus): a source-filter theory approach. Scientific Reports 5:17255. DOI: 10.1038/srep17255.

Favaro L., Ozella L., Pessani D. 2014. The Vocal Repertoire of the African Penguin (Spheniscus demersus): Structure and Function of Calls. PLoS ONE 9:e103460. DOI: 10.1371/journal.pone.0103460.

Fox J., Weisberg S. 2011. Multivariate linear models in R. In: An R companion to applied regression. SAGE Publications,.

Friard O., Gamba M. 2016. BORIS: a free, versatile open-source event-logging software for video/audio coding and live observations. Methods in Ecology and Evolution 7:1325-1330. DOI: 10.1111/2041$210 X .12584$.

Gómez J-C. 2005. Species comparative studies and cognitive development. Trends in Cognitive Sciences 9:118-125. DOI: 10.1016/j.tics.2005.01.004.

Hare B., Call J., Agnetta B., Tomasello M. 2000. Chimpanzees know what conspecifics do and do not see. Animal behaviour 59:771-785. DOI: 10.1006/anbe.1999.1377.

Hopper LM. 2017. Cognitive research in zoos. Current Opinion in Behavioral Sciences 16: 100-110. DOI: 10.1016/j.cobeha.2017.04.006

Kaminski J., Riedel J., Call J., Tomasello M. 2005. Domestic goats, Capra hircus, follow gaze direction and use social cues in an object choice task. Animal Behaviour 69:11-18. DOI: 10.1016/j.anbehav.2004.05.008.

Kehmeier S., Schloegl C., Scheiber IBR., Weiß BM. 2011. Early development of gaze following into distant space in juvenile Greylag geese (Anser anser). Animal Cognition 14:477-485. DOI: 
10.1007/s10071-011-0381-x.

324

Kummer H., Daston L., Gigerenzer G., Silk J. 1997. The social intelligence hypothesis. In: Weingart P, Richerson P, Mitchell SD, Maasen S eds. Human by Nature: Between Biology and the Social Sciences. Hillsdale, New Jersey: Lawrence Erlbaum, 157-179.

Loretto M-C., Schloegl C., Bugnyar T. 2010. Northern bald ibises follow others' gaze into distant space but not behind barriers. Biology letters 6:14-7. DOI: 10.1098/rsbl.2009.0510.

MacLean EL., Sandel A., Bray J., Oldenkamp RE., Reddy RB., Hare B. 2013. Group Size Predicts Social but Not Nonsocial Cognition in Lemurs. PLoS ONE 8:e66359. DOI: 10.1371/journal.pone.0066359.

Martin GR. 2009. What is binocular vision for? A birds' eye view. Journal of Vision 9:14-14. DOI:

$$
\text { 10.1167/9.11.14. }
$$

Overduin-de Vries a M., Spruijt BM., Sterck EHM. 2013. Long-tailed macaques (Macaca fascicularis) understand what conspecifics can see in a competitive situation. Animal cognition. DOI: 10.1007/s10071-013-0639-6.

Ozella L., Favaro L., Carnovale I., Pessani D. 2015. Pond Use by Captive African Penguins (Spheniscus demersus) in an Immersive Exhibit Adjacent to Human Bathers. Journal of Applied Animal Welfare Science 18:303-309. DOI: 10.1080/10888705.2014.977384.

Pichegru L. 2013. Increasing breeding success of an Endangered penguin: artificial nests or culling predatory gulls? Bird Conservation International 23:296-308. DOI: 10.1017/S0959270912000135.

Rosati AG., Hare B. 2009. Looking past the model species: diversity in gaze-following skills across primates. Current Opinion in Neurobiology 19:45-51. DOI: 10.1016/j.conb.2009.03.002.

Sandel AA., MacLean EL., Hare B. 2011. Evidence from four lemur species that ringtailed lemur social cognition converges with that of haplorhine primates. Animal Behaviour 81:925-931. DOI: 10.1016/j.anbehav.2011.01.020.

Schloegl C., Kotrschal K., Bugnyar T. 2007. Gaze following in common ravens, Corvus corax: ontogeny and habituation. Animal Behaviour 74:769-778. DOI: 10.1016/j.anbehav.2006.08.017.

Seed A., Emery N., Clayton N. 2009. Intelligence in Corvids and Apes: A Case of Convergent Evolution? Ethology 115:401-420. DOI: 10.1111/j.1439-0310.2009.01644.x.

Shettleworth SJ. 2009. The evolution of comparative cognition: Is the snark still a boojum? Behavioural 
$351 \quad$ Processes 80:210-217.

352 Sutton GJ., Hoskins AJ., Arnould JPY. 2015. Benefits of Group Foraging Depend on Prey Type in a Small 353 Marine Predator, the Little Penguin. PloS ONE 10:e0144297. DOI: 10.1371/journal.pone.0144297. 354 Tomasello M., Call J., Hare B. 1998. Five primate species follow the visual gaze of conspecifics. Animal 355 Behaviour 55:1063-1069.

356 Werhahn G., Virányi Z., Barrera G., Sommese A., Range F. 2016. Wolves (Canis lupus) and dogs (Canis 357 familiaris) differ in following human gaze into distant space but respond similar to their packmates' 358 gaze. Journal of Comparative Psychology 130:288-298. DOI: 10.1037/com0000036.

359 Wilkinson A., Mandl I., Bugnyar T., Huber L. 2010. Gaze following in the red-footed tortoise (Geochelone 360 carbonaria). Animal Cognition 13:765-769. DOI: 10.1007/s10071-010-0320-2.

361 Williams TD. 1995. The Penguins. Oxford: Oxford University Press.

362 Young E. 2005. Skua and Penguin: Predator and Prey. Cambridge University Press. 363 